\title{
Dento-facial aesthetics in the eyes of adolescent patients during the Covid-19 pandemic.
}

\author{
Jessica Olivia Cherecheș ${ }^{1}$, Abel Emanuel Moca ${ }^{1}$, Luminița Ligia Vaida ${ }^{1}$, Marius Bembea ${ }^{2}$ \\ ${ }^{1}$ University of Oradea, Department of Dentistry, Faculty of Medicine and Pharmacy, Romania. \\ ${ }^{2}$ University of Oradea, Department of Preclinical Disciplines, Faculty of Medicine and Pharmacy, Romania.
}

\begin{abstract}
Introduction: Dental malocclusions are considered a major issue in adolescence, and are often the main reason for which patients go to the dentist. Adolescents' option to start an orthodontic treatment is questioned in the light of the restrictions imposed in the context of the Covid-19 pandemic.

Aim of the study: The aim of this study was to assess adolescents' perceptions of facial aesthetics and the impact of the Covid-19 pandemic on the decision to start an orthodontic treatment during this time, when a number of restrictions, such as face mask wearing, were imposed.

Material and methods: A 15 items questionnaire was applied in the city of Oradea, Romania and distributed to orthodontic adolescent patients, with a previously diagnosed malocclusion, with ages between 12 and 17.9 years. The questionnaires were correctly filled in by 76 participants.

Results: Patients tend to be affected by the aspect of their teeth (50\%), and most patients considered that the aspect of their teeth forces them to socialize less (44.8\%), and to avoid smiling (44.8\%). $39.4 \%$ of the respondents considered that wearing a face mask is useful because it covers all dental malocclusions, and $31.6 \%$ of the respondents were not bothered that they had to wear a face mask.

Conclusions: Patients declared that they were affected by the aspect of their teeth, and have a generally positive attitude regarding face masks.

Keywords: malocclusions, face masks, Covid-19, adolescents.
\end{abstract}

\section{Introduction}

Dental anomalies occur as a result of the complex interaction of some genetic and environmental factors [1] that intervene and cause disturbances in the processes of morphodifferentiation or histo-differentiation of dental units [2]. Occlusal features, type and frequency of dental anomalies influence the sagittal ratio between dental arches [3]. These congenital defects manifest in various clinical forms [4], with different degrees of severity [5]. From a functional point of view, dental anomalies due to the malocclusion they cause, favor disorders of mastication, swallowing, phonation, and to a significant extent, change the patient's physiognomy [5].

At a young age, social discrimination due to the disharmonious facial appearance, including oral aesthetics, has negative effects on the quality of life of the individual, and causes physical, psychological and social disturbances [6], the adolescent perceiving this as a stigma $[7,8]$.
Dental malocclusions are considered a major issue in adolescence, dental anomalies that negatively influence facial aesthetics often being the main reason for which patients go to the dentist [9]. The treatment of malocclusions is performed with the help of orthodontic appliances. This method of treatment is increasingly accepted, and it is considered as a solution to the problems of facial aesthetics and social reintegration [10].

The Covid-19 pandemic manifested by a severe acute respiratory syndrome, and caused by the new coronavirus (SARS-CoV-2), has been described by the World Health Organization as a new form of viral pneumonia [11].

Adolescents' option to start an orthodontic treatment, justified by the need to correct any diagnosed malocclusion and the desire to increase self-confidence [12] is questioned in the light of the restrictions imposed in the context of the Covid-19 pandemic. There was, also, a period in which dental offices' activity was interrupted, which could have had a 
negative influence on the evolution of the orthodontic treatment [13].

\section{Aim of the study}

The aim of this study was to assess adolescents' perceptions of facial aesthetics and the impact of the Covid-19 pandemic on the decision to start an orthodontic treatment during this time, when a number of restrictions were imposed. The attitude of the patients regarding the compulsoriness of protective face mask wearing was investigated as well.

\section{Material and methods}

\section{Participants and Data Collection}

The study was carried out over a period of two months, between February 2021 and March 2021. During this period, restrictive measures regarding the mandatory wearing of face masks and social distancing were active.

The authors conceived a questionnaire consisting of 15 items. The questionnaires were printed on paper and applied in two private orthodontic practices from Oradea, Romania.
They were distributed to adolescent patients, aged between 12 and 17.9 years, both girls and boys, from urban and rural areas. The respondents were adolescent patients with previously diagnosed dento-maxillary anomalies who had received the indication for orthodontic treatment. Before filling in the questionnaires, all patients were informed that they were applied for research purposes, and that by filling in the questionnaires, they confirmed their willingness to participate anonymously in this study. Patients had the possibility to withdraw from the research with no consequences. No financial benefits were promised to the respondents. No time limit was imposed. The language used for the questionnaires was Romanian.

A Likert-type scale was used for all items. Participants had to choose a number from 0 to 5 ( 0 being the lowest possible score and meaning a complete negation, and 5 being the highest possible score and meaning a complete affirmation) in order to assess their attitude regarding the question. Items are translated in Table 1.

Table 1. Items used in the questionnaires

\begin{tabular}{ll}
\hline Item & \multicolumn{1}{c}{ Question } \\
\hline Item 1 & "Are you bothered by the aspect of your face?" \\
Item 2 & "Are you bothered by the aspect of your teeth?" \\
Item 3 & "Do you believe that the aspect of your teeth influences the aspect of your face?" \\
Item 4 & "Do you believe that the aspect of your teeth negatively affects the relationship with other \\
Item 5 & "Deople?" \\
Item 6 & "Does the aspect of your teeth makes you socialize less?" \\
Item 7 & "Are you trying to hide your teeth when you speak, smile or laugh?" \\
Item 8 & "Do you want to have the aspect of your teeth improved?" \\
Item 9 & "Do you still want to have the aspect of your teeth corrected in the context of the Covid-19 \\
Item 10 & "Does face mask help in hiding your teeth?" \\
Item 11 & "Are you concerned about face mask wearing?" \\
Item 12 & "Are you sad because you have to wear a face mask?" \\
Item 13 & "Are you happy because you have to wear a face mask?" \\
Item 14 & "Do you believe that wearing a fixed orthodontic appliance will make you look bad?" \\
Item 15 & "Do you believe that now, when face mask wearing is mandatory, is a good time to start the \\
\end{tabular}

The following inclusion criteria were applied: adolescent patients with dentomaxillary anomalies and the indication of a fixed orthodontic appliance bonded on the labial surfaces of the teeth, with ages between
12 and 17.9 years, living in Romania. The questionnaires belonging to patients who answered incompletely or incorrectly were excluded from the study. Incomplete answered questionnaires were survey forms where only a 
part of the questions received an answer, while incorrectly answered questionnaires were survey forms where more than one answer was provided for one or more questions.

The questionnaires were handed out to a number of 98 adolescent patients with dentomaxillary anomalies, but only 85 agreed to take part in this research and filled in the survey forms. After applying the exclusion criteria, a number of 76 participants remained in the study.

\section{Statistical Analysis}

The statistical analysis was performed by using IBM SPSS software, version 25 (IBM, Chicago, IL, USA). Quantitative variables were tested for distribution using the Shapiro-Wilk test and were expressed as mean values with standard deviations or medians with interpercentile intervals. The independent quantitative variables with a non-parametric distribution were tested with the MannWhitney $\mathrm{U}$ test, and all correlations between them were verified with the Spearman's rho correlation coefficient. Qualitative variables were expressed as absolute numbers or percentages.

\section{Ethical Considerations}

The study was conducted in accordance with the 1964 Declaration of Helsinki and its later amendments and was approved by the
Research Ethics Committee of the University of Oradea.

\section{Results}

Socio-Demographic Data

The mean age of the participants was 15.03 \pm 1.65 years with a median of 15 years, and a range between 12 and 17.9 years. Of all the respondents, 50 were girls $(65.8 \%)$ and 26 were boys (34.25). Regarding the living environment of the participants, 26 patients were from a rural environment (34.25) and 50 were from an urban environment $(65.8 \%)$.

Answers

The responses given for all the items are summarized in Table 2 . The answers provided for item 2 showed that patients tend to be affected by the aspect of their teeth, scores of 4 and 5 representing half of the total answers given. Most of the patients considered that the aspect of their teeth forces them to socialize less (Item 5), and to avoid smiling (Item 7). In regards to their desire to correct the position of their teeth, the majority of the respondents wanted to correct the position of their teeth (Item 8), and this desire was not affected by the Covid-19 pandemic. $39.4 \%$ of the respondents considered that wearing a face mask is useful because it covers all dental malocclusions, and $31.6 \%$ of the respondents were not bothered that they had to wear a face mask.

Table 2. Answers provided by the participants

\begin{tabular}{|c|c|c|c|c|c|c|}
\hline $\begin{array}{l}\text { Answer } \\
\text { (No., \%) }\end{array}$ & 0 & 1 & 2 & 3 & 4 & 5 \\
\hline Item 1 & $21.1 \%$ & $26.3 \%$ & $28.9 \%$ & $7.9 \%$ & $15.8 \%$ & $0 \%$ \\
\hline Item 2 & $2.6 \%$ & $13.2 \%$ & $28.9 \%$ & $5.3 \%$ & $34.2 \%$ & $15.8 \%$ \\
\hline Item 3 & $10.5 \%$ & $21.1 \%$ & $21.1 \%$ & $7.9 \%$ & $31.6 \%$ & $7.9 \%$ \\
\hline Item 4 & $0 \%$ & $5.3 \%$ & $42.1 \%$ & $34.2 \%$ & $13.2 \%$ & $5.3 \%$ \\
\hline Item 5 & $0 \%$ & $13.2 \%$ & $42.1 \%$ & $15.8 \%$ & $21.1 \%$ & $7.9 \%$ \\
\hline Item 6 & $21.1 \%$ & $13.2 \%$ & $36.8 \%$ & $5.3 \%$ & $21.1 \%$ & $2.6 \%$ \\
\hline Item 7 & $0 \%$ & $10.5 \%$ & $39.5 \%$ & $5.3 \%$ & $39.5 \%$ & $5.3 \%$ \\
\hline Item 8 & $0 \%$ & $5.3 \%$ & $7.9 \%$ & $0 \%$ & $39.5 \%$ & $47.4 \%$ \\
\hline Item 9 & $0 \%$ & $0 \%$ & $5.3 \%$ & $7.9 \%$ & $23.7 \%$ & $63.2 \%$ \\
\hline Item 10 & $0 \%$ & $18.4 \%$ & $28.9 \%$ & $13.2 \%$ & $36.8 \%$ & $2.6 \%$ \\
\hline Item 11 & $28.9 \%$ & $15.8 \%$ & $23.7 \%$ & $13.2 \%$ & $13.2 \%$ & $5.3 \%$ \\
\hline Item 12 & $0 \%$ & $15.8 \%$ & $31.6 \%$ & $23.7 \%$ & $23.7 \%$ & $5.3 \%$ \\
\hline Item 13 & $0 \%$ & $7.9 \%$ & $13.2 \%$ & $23.7 \%$ & $39.5 \%$ & $15.8 \%$ \\
\hline Item 14 & $60.5 \%$ & $10.5 \%$ & $23.7 \%$ & $5.3 \%$ & $0 \%$ & $0 \%$ \\
\hline Item 15 & $0 \%$ & $5.3 \%$ & $26.3 \%$ & $5.3 \%$ & $50 \%$ & $13.2 \%$ \\
\hline
\end{tabular}

No.: Number; \%: percentage 
Correlative and comparative results

Statistically significant correlations were found between the age of the respondents and answers given for Items 2, 6, 11 and 12. These are detailed in Table 3. As such, older patients were more confident with the aspect of their teeth (Item 2) and were less stressed by the aspect of their teeth (Item 6). However, when it comes to wearing the protective face masks, older patients were more concerned about the fact that they had to wear a face mask (Item 11), and were less happy about face mask wearing (Item 12).

Table 3. Correlation between age and Items 2, 6, 11, 12

\begin{tabular}{cc}
\hline Correlation & $p^{*}$ \\
\hline Age $\left(p<0.001^{* *}\right) \times$ Item $2\left(p<0.001^{* *}\right)$ & $0.003, R=0.341$ \\
Age $\left(p<0.001^{* *}\right) \times$ Item $6\left(p<0.001^{* *}\right)$ & $0.046, R=-0.230$ \\
Age $\left(p<0.001^{* *}\right) \times$ Item $11\left(p<0.001^{* *}\right)$ & $0.035, R=0.242$ \\
Age $\left(p<0.001^{* *}\right) \times$ Item $12\left(p<0.001^{* *}\right)$ & $0.037, R=-0.240$ \\
\hline
\end{tabular}

*: Spearman's rho Correlation Coefficient; ${ }^{* *}$ : Shapiro-Wilk Test

Table 4 shows the statistically significant comparisons identified in the studied sample, in relation to respondents' gender. In the studied sample, boys are more affected by the aspect of their teeth than girls (Item 2), and are more stressed by this matter (Item 6). Boys, more than girls, considered that face mask is useful in covering dental malocclusions (Item $10)$.

Table 4. Comparisons between answers provided for Items 2, 6, 10 in relation to patients' gender

\begin{tabular}{|c|c|c|c|c|}
\hline Gender & Mean value \pm SD & Median (IQR) & Medium Rank & $p^{*}$ \\
\hline \multicolumn{5}{|c|}{ Item 2} \\
\hline Girls $\left(p<0.001^{* *}\right)$ & $2.88 \pm 1.189$ & $3(2-4)$ & 42.06 & 0.041 \\
\hline Boys $\left(p<0.001^{* *}\right)$ & $2.31 \pm 1.087$ & $2(1.75-3.25)$ & 31.65 & \\
\hline \multicolumn{5}{|c|}{ Item 6} \\
\hline Girls $\left(p<0.001^{* *}\right)$ & $1.72 \pm 1.578$ & $2(0-3)$ & 34.06 & 0.012 \\
\hline Boys $\left(p<0.001^{* *}\right)$ & $2.54 \pm 1.029$ & $2(2-4)$ & 47.04 & \\
\hline \multicolumn{5}{|c|}{ Item 10} \\
\hline Girls $\left(p<0.001^{* *}\right)$ & $2.56 \pm 1.181$ & $2(2-4)$ & 34.86 & 0.037 \\
\hline Boys $\left(p<0.001^{* *}\right)$ & $3.15 \pm 1.19$ & $4(2-4)$ & 45.50 & \\
\hline
\end{tabular}

SD: Standard Deviation; IQR: Interquartile Range; *: Mann-Whitney U Test; **: Shapiro-Wilk Test

Table 5 shows the statistically significant comparisons identified in the studied sample, in relation to patients' living environment. Patients from the rural environment are more affected by the aspect of their teeth (Item 2) in comparison with patients from an urban environment. Patients living in an urban environment considered the orthodontic treatment less necessary (Item 8) than patients living in a rural environment. Face mask wearing was considered more useful for covering dental malocclusions by patients living in an urban environment (Item 10), caused less concern for these category of patients (Item 11), and more happiness for patients living in an urban environment in comparison with patients living in a rural environment (Item 12).

Table 5. Comparisons between answers provided for Items 2, 8, 10, 11, 12 in relation to patients' living environment

\begin{tabular}{lcccc}
\hline \multicolumn{1}{c}{ Living environment } & Mean value \pm SD & Median (IQR) & Medium Rank & $p^{*}$ \\
\hline & & Item 2 & 0.037 \\
Rural $\left(p=0.025^{* *}\right)$ & $3.08 \pm 1.23$ & $3(2-4)$ & 45.50 & 34.86 \\
Urban $\left(p<0.001^{* *}\right)$ & $2.48 \pm 1.11$ & $2(2-3.25)$ & & 0.008 \\
& & Item 8 & 46.96 & \\
Rural $\left(p<0.001^{* *}\right)$ & $4.54 \pm 0.859$ & $5(4-5)$ & 34.10 & \\
Urban $\left(p<0.001^{* *}\right)$ & $3.96 \pm 1.195$ & $4(4-5)$ & Item 10 & \\
& & &
\end{tabular}




\begin{tabular}{|c|c|c|c|c|}
\hline Rural $\left(p<0.001^{* *}\right)$ & $2.38 \pm 1.299$ & $2(1-4)$ & 31.81 & 0.047 \\
\hline Urban $\left(p<0.001^{* *}\right)$ & $2.96 \pm 1.124$ & $3(2-4)$ & 41.98 & \\
\hline \multicolumn{5}{|c|}{ Item 11} \\
\hline Rural $\left(p<0.001^{* *}\right)$ & $1.38 \pm 1.675$ & $1(0-2.25)$ & 31.73 & 0.049 \\
\hline Urban $\left(p=0.002^{* *}\right)$ & $2.04 \pm 1.47$ & $2(1-3)$ & 42.02 & \\
\hline \multicolumn{5}{|c|}{ Item 12} \\
\hline Rural $\left(p=0.015^{* *}\right)$ & $2.77 \pm 1.07$ & $3(2-3.25)$ & 25.58 & $<0.001$ \\
\hline Urban $\left(p<0.001^{* *}\right)$ & $3.76 \pm 1.04$ & $4(3-4)$ & 45.22 & \\
\hline
\end{tabular}

SD: Standard Deviation; IQR: Interquartile Range; ${ }^{*}$ : Mann-Whitney U Test; ${ }^{* *}$ : Shapiro-Wilk Test

Statistically significant correlations were identified between different items. As such, patients that were more affected by the aspect of their face were happier to wear a face mask (Item 1 and Item 13), as were patients that were more affected by the aspect of their teeth (Item 2 and Item 13). Patients who considered that the aspect of the teeth influences the aspect of the face considered the Covid-19 pandemic to be an untimely moment to start an orthodontic treatment because face mask would cover the orthodontic appliance (Item 3 and Item 15). Patients who considered that the aspect of their teeth negatively impacts their social life were happier to wear a face mask (Item 4 and Item 13). Participants who said that they want to have their teeth' position corrected as fast as possible were less worried that they had to wear a face mask (Item 8 and Item 11). The identified statistically significant correlations are presented in Table 6 .

Table 6. Correlations between items

\begin{tabular}{|c|c|}
\hline Correlations & $p^{*}$ \\
\hline Item $1\left(p<0.001^{* *}\right) \times$ Item $13\left(p<0.001^{* *}\right)$ & $0.005, R=0.319$ \\
\hline Item $2\left(p<0.001^{* *}\right) \times$ Item $13\left(p<0.001^{* *}\right)$ & $0.004, R=0.330$ \\
\hline Item $3\left(p<0.001^{* *}\right) \times$ Item $12\left(p<0.001^{* *}\right)$ & $<0.001, R=-0.391$ \\
\hline Item $3\left(p<0.001^{* *}\right) \times$ Item $13\left(p<0.001^{* *}\right)$ & $0.002, R=0.354$ \\
\hline Item $3\left(p<0.001^{* *}\right) \times$ Item $15\left(p<0.001^{* *}\right)$ & $0.007, R=-0.306$ \\
\hline Item $4\left(p<0.001^{* *}\right) \times$ Item $13\left(p<0.001^{* *}\right)$ & $0.013, R=0.284$ \\
\hline Item $4\left(p<0.001^{* *}\right) \times$ Item $15\left(p<0.001^{* *}\right)$ & $0.025, R=-0.257$ \\
\hline Item $5\left(p<0.001^{* *}\right) \times$ Item $13\left(p<0.001^{* *}\right)$ & $<0.001, R=-0.398$ \\
\hline Item $6\left(p<0.001^{* *}\right) \times$ Item $9\left(p<0.001^{* *}\right)$ & $0.040, R=-0.236$ \\
\hline Item $6\left(p<0.001^{* *}\right) \times$ Item $12\left(p<0.001^{* *}\right)$ & $0.050, R=-0.226$ \\
\hline Item $6\left(p<0.001^{* *}\right) \times$ Item $13\left(p<0.001^{* *}\right)$ & $0.030, R=0.249$ \\
\hline Item $7\left(p<0.001^{* *}\right) \times$ Item $13\left(p<0.001^{* *}\right)$ & $<0.001, R=0.504$ \\
\hline Item $8\left(p<0.001^{* *}\right) \times$ Item $11\left(p<0.001^{* *}\right)$ & $0.034, R=-0.243$ \\
\hline Item $8\left(p<0.001^{* *}\right) \times$ Item $12\left(p<0.001^{* *}\right)$ & $0.010, R=-0.294$ \\
\hline Item $8\left(p<0.001^{* *}\right) \times$ Item $15\left(p<0.001^{* *}\right)$ & $0.008, R=-0.303$ \\
\hline Item $9\left(p<0.001^{* *}\right) \times$ Item $13\left(p<0.001^{* *}\right)$ & $<0.001, R=-0.395$ \\
\hline Item $9\left(p<0.001^{* *}\right) \times$ Item $14\left(p<0.001^{* *}\right)$ & $<0.001, R=-0.412$ \\
\hline Item $10\left(p<0.001^{* *}\right) \times$ Item $13\left(p<0.001^{* *}\right)$ & $<0.001, R=0.417$ \\
\hline Item $12\left(p<0.001^{* *}\right) \times$ Item $14\left(p<0.001^{* *}\right)$ & $0.040, R=0.236$ \\
\hline
\end{tabular}

*: Spearman's rho Correlation Coefficient; ${ }^{* *}$ : Shapiro-Wilk Test

\section{Discussions}

The Covid-19 pandemic represents a recent crisis with serious worldwide effects, which imposed the need for a lockdown, in an attempt to limit the spread of the disease [14]. During the lockdown period, dental offices had their activity suspended [15], which caused stress and anxiety for orthodontic patients [14]. Patients that were previously diagnosed with a dental malocclusion were targeted in this survey. The aim was to find out what was their opinion about the existing malocclusion and how beneficial did they find face mask wearing to be, given their malocclusion.

In order to be able to objectively assess the concerns and attitudes regarding face mask wearing, as a mandatory measure, we chose to apply questionnaires to adolescent patients 
with dento-maxillary anomalies. The necessity for an orthodontic treatment was established prior to the onset of the Covid-19 pandemic for all these patients. Questionnaires are considered a valid method that can be used in order to analyze patients' opinions regarding a specific topic [16].

In the vast majority of studies conducted during the Covid-19 pandemic, and especially during lockdown, the questionnaires were applied online, through platforms [17,18], websites [19,20] or e-mail addresses [21], but also in a combined version of both online and on paper [22]. However, in this study, the questionnaires were applied in the orthodontic office after resuming dental offices' activity, in order to evaluate the perception of adolescent patients on the lockdown period and to understand exactly what they wanted, as well as which were their dissatisfactions in the current context. In addition, any ambiguities in the formulation of the questions could be easily clarified, but without influencing their answers and without promising them certain rewards.

As expected, the results obtained showed that patients who were more disturbed by the aspect of their teeth or face were more happy to wear a protective face mask. The reason for that could be that face mask covers the lower part of the face, and, therefore, covers any visible malocclusion. Face mask wearing was perceived as an aid in developing better social contacts by patients who considered that the aspect of their teeth negatively impacts their social life.

Age had an important influence on the answers provided for some items. Older patients were more confident with the aspect of their teeth, but when it comes to wearing the protective face masks, older patients were more concerned about the fact that they had to wear a face mask, and were less happy about face mask wearing. This could be due to adolescents tendency to disobey any rules imposed by adults, and due to commodity.

The living environment of the patients impacted the way they answered. Patients living in an urban environment considered face mask wearing for covering malocclusions to be more useful, and it caused less concern for them. Interestingly enough, gender influenced answers in an unexpected manner. Boys, more than girls, were more negatively affected and were more stressed by the aspect of their teeth.

Comparing the results obtained in this research with other studies is not possible because there has been no identified survey that was applied on adolescent orthodontic patients, and that investigated the attitude towards face mask wearing. This is the novelty of this study. It focuses on a specific group of patients, adolescent orthodontic patients with a diagnosed dental malocclusion, and investigates their opinion on dento-facial aesthetics and face mask wearing in the Covid19 pandemic.

The study has its limitations. The size of the sample could be larger for more conclusive results, and the online application of the questionnaires would permit a national approach of this topic. However, we consider it to be a solid starting point for future research.

\section{Conclusions}

In general, patients declared that they were affected by the aspect of their teeth, and boys, more than girls, were affected by this matter. In the studied sample, patients have a generally positive attitude regarding face masks and consider face mask wearing as an aid for covering dental malocclusions.

Conflict of interest: None to declare.

\section{Acknowledgments}

This research was funded through the Project 123008, entitled "SmartDoct - High quality programs for doctoral students and postdoctoral researchers from the University of Oradea for growth of the relevance of research and innovation in the context of regional economy", project financed through the Human Capital Operation Program 20142020 .

\section{References}

1. Brook AH, Griffin RC, Townsend G, Levisianos I, Russell J, Smith RN. Variability and patterning in permanent tooth size of four human ethnic groups. Arch Oral Biol. 2009; 54:79-85.

2. Laganà G, Venza N, Borzabadi-Farahani A, Fabi F, Danesi C, Cozza P. Dental anomalies: prevalence and associations between them in a large sample 
of non-orthodontic subjects, a cross-sectional study. BMC Oral Health. 2017; 17:62.

3. Joseffson E, Bjerklin K, Lindsten R. Malocclusion frequency in Swedish and immigrant adolescents: influence of origin on orthodontic treatment need. European J of Orthod. 2007; 29:79-87.

4. Roslan AA, Rahman NA, Alam MK. Dental anomalies and their treatment modalities/planning in orthodontic patients. J Orthod Sci. 2018; 7:16.

5. Fernandez CCA, Pereira CVCA, Luiz RR, Vieira AR, De Castro Costa M. Dental anomalies in different growth and skeletal malocclusion patterns. Angle Orthod. 2018; 88:195-201.

6. Scheffel DLS, Jeremias F, Fragelli CMB, Dos SantosPinto LAM, Hebling J, de Oliveira OB Jr. Esthetic dental anomalies as motive for bullying in schoolchildren. Eur J Dent. 2014; 8:124-128.

7. Cunningham SJ. The psychology of facial appearance. Dent Update. 1999; 26:438-43.

8. Perillo L, Esposito M, Caprioglio A, Attanasio S, Santini AC, Carotenuto M. Orthodontic treatment need for adolescents in the Campania region: the malocclusion impact on self-concept. Patient Prefer Adherence. 2014; 8:353-359.

9. Nobile CG, Pavia M, Fortunato L, Angelillo IF. Prevalence and factors related to malocclusion and orthodontic treatment need in children and adolescents in Italy. Eur J Public Health. 2007; 17:637-41.

10. Klages U, Erbe C, Sandru SD, Brüllman D, Wehrbein H. Psychosocial impact of dental aesthetics in adolescence: validity and reliability of a questionnaire across age-groups. Qual Life Res. 2015; 24:379-390.

11. Bahramian H, Gharib B, Baghalian A. COVID-19 Considerations in Pediatric Dentistry. JDR Clin Trans Res. 2020; 5:307-311.

12. Liu Z, McGrath C, Hägg $U$. The impact of malocclusion/orthodontic treatment need on the quality of life. A systematic review. Angle Orthod. 2009; 79:585-91.
13. Shenoi SB, Deshpande S, Jatti R. Impact of COVID19 Lockdown on Patients Undergoing Orthodontic Treatment: A Questionnaire Study. J Indian Orthod Soc. 2020; 54:195-202.

14. Saeed S, Awasthi AA, Nandi D, Kaur K, Hasan S, Janardhanan R. Knowledge, attitude and practice towards COVID-19 among individuals with associated comorbidities. J of Medicine and Life. 2021; 14:225-237.

15. Morosan H. Orthodontic treatment in times of Covid-19. J of Medicine and Life. 2021; 14:205209.

16. Cagetti MG, Cairoli JL, Senna A, Campus G. COVID19 Outbreak in North Italy: An Overview on Dentistry. A Questionnaire Survey. Int J Environ Res Public Health. 2020; 17:3835.

17. Carvalho JC, Declerck D, Jacquet W, Bottenberg P. Dentist Related Factors Associated with Implementation of COVID-19 Protective Measures: A National Survey. Int. J. of Environ. Res and Public Health. 2021; 18:8381.

18. Cotrin P, Peloso RM, Oliveira RC, et al. Impact of coronavirus pandemic in appointments and anxiety/concerns of patients regarding orthodontic treatment. Orthod Craniofac Res. 2020; 23:455-461.

19. Xiong $X, W u Y$, Fang $X$ et al. Mental distress in orthodontic patients during the coronavirus disease 2019 pandemic. Am J Orthod Dentofacial Orthop. 2020; 158:824-833.

20. Stylianou N, Samouti G, Samoutis G. Mental Health Disorders During the COVID-19 Outbreak in Cyprus. J of Medicine and Life. 2020; 13:300-305.

21. Schmidt J, Waldova E, Balkova S, Suchanek J, Smucler R. Impact of COVID-19 on Czech Dentistry: A Nationwide Cross-Sectional Preliminary Study among Dentists in the Czech Republic. Int J Environ Res Public Health. 2021; 18:9121.

22. Habib MA, Dayyab FM, Iliyasu G, Habib AG. Knowledge, attitude and practice survey of COVID19 pandemic in Northern Nigeria. PLoS One. 2021; 16:e0245176.

\section{Corresponding author:}

Abel Emanuel Moca

Department of Dentistry, Faculty of Medicine and Pharmacy, University of Oradea, 10 Piața 1 Decembrie Street, 410073 Oradea, Romania.

E-mail: abelmoca@yahoo.com 